\title{
Importance of the phase and amplitude in the fractional Fourier domain
}

\author{
Tatiana Alieva and Maria Luisa Calvo \\ Departamento de Optica, Facultad de Ciencias Fisicas, Universidad Complutense de Madrid, 28040 Madrid, Spain
}

Received June 14, 2002; revised manuscript received October 1, 2002; accepted October 2, 2002

\begin{abstract}
The importance of the amplitude and phase in the fractional Fourier transform (FT) domain is analyzed on the basis of the rectangular signal and the real-world image. The quality of signal restoration from only the amplitude or from only the phase of its fractional FT by applying the inverse fractional FT is considered. It is shown that the signal reconstructed from the amplitude of the fractional FT usually reveals the main features of the original signal only for relatively low fractional orders. On the basis of phase information in the fractional FT domains, significant details of the signal can be obtained for nearly all fractional orders. () 2003 Optical Society of America
\end{abstract}

OCIS codes: 100.5090, 100.3010, 070.6110.

\section{INTRODUCTION}

Nowadays the performance of conventional correlation and convolution operations in optics is usually based on the implementation of phase-only filters. ${ }^{1-3}$ Application of correlation and convolution operations in the fractional Fourier domains for pattern recognition, noise reduction, etc., has recently been proposed..$^{4-10}$ This requires investigation of the importance of phase and amplitude in the fractional Fourier domains.

The same question has been addressed for the ordinary Fourier domain in many publications; see, for example, Refs. 11-14. In the most recent paper Lohmann et al. ${ }^{14}$ concluded that "there is no strong confirmation of the phase dominance and that the signal symmetry and reality play an important role in determining the significance of amplitude and phase in the Fourier domain." We agree with this statement and will show that it can be extended to the case of the fractional FT domain. Several examples in Section 2 clearly demonstrate the dominance of the phase or the amplitude.

From another point of view, the reconstruction of realworld images from the phase of their FTs leads to edge enhancement $^{11-13}$ because elimination of the FT amplitude information stresses the high frequencies. Therefore, the form of the image very often can be detected from the phase of the FT.

In this paper we study the question of signal reconstruction from the amplitude or from the phase of its fractional FT. As an object for analysis we have chosen a rectangular signal, which has clearly defined edges and whose form is detectable from only the phase data of the FT. We show how the slight dominance of amplitude information for low fractional orders is transformed into phase dominance for the higher ones. This tendency also holds for real-world images as is demonstrated in the example of the "cameraman" photograph.

\section{SOME EXAMPLES OF PHASE OR AMPLITUDE DOMINANCE IN THE FRACTIONAL FOURIER DOMAIN}

The fractional FT is a generalization of the ordinary FT. $^{10,15-17}$ Its kernel depends on the parameter $\alpha$, related to the fractional order $p$ as $\alpha=\pi p / 2$, and which can be interpreted as a rotation angle in the phase space. The fractional FT of a function $f(x)$ for angle $\alpha$ is defined as

$$
R^{\alpha}[f(x)](u)=F_{\alpha}(u)=\int f(x) K(\alpha, x, u) \mathrm{d} x
$$

where the kernel is given by

$$
K(\alpha, x, u)=\frac{\exp (i \alpha / 2)}{(i \sin \alpha)^{1 / 2}} \exp \left[i \pi \frac{\left(x^{2}+u^{2}\right) \cos \alpha-2 x u}{\sin \alpha}\right]
$$

Thus for $\alpha=0$ it corresponds to the identity transform; for $\alpha=\pi / 2(p=1)$ and $\alpha=3 \pi / 2$ it reduces to the FT and the inverse FT, respectively. Moreover, $F_{\pi}(u)$ $=f(-u)$. The kernel of the fractional $\mathrm{FT}$ is periodic on $\alpha$. It is easy to see from Eq. (2) that $K^{*}(\alpha, x, u)$ $=K(-\alpha, x, u)$.

The fractional FT describes, in the paraxial approximation, the propagation of optical beams through a medium with quadratic refractive index. The fractional FT of a complex wave amplitude $f(x)$ can be performed by using different optical setups discussed, for example, in Refs. 5 and 10.

In the context of the paper it is convenient to represent the fractional FT in the form 


$$
\begin{aligned}
F_{\alpha}(u) \equiv & \left|F_{\alpha}(u)\right| \exp \left[i 2 \pi \varphi_{\alpha}(u)\right], \\
\equiv & \frac{1}{\sqrt{\sin \alpha}} \exp \left(i \pi u^{2} \cot \alpha+i \alpha / 2-i \pi / 4\right) \\
& \quad \times \int f(x) \exp \left(i \pi \frac{x^{2} \cos \alpha-2 x u}{\sin \alpha}\right) \mathrm{d} x, \\
\equiv & \frac{1}{\sqrt{\sin \alpha}} \exp \left(i \pi u^{2} \cot \alpha+i \alpha / 2-i \pi / 4\right) \\
& \quad \times A_{\alpha}(u) \exp \left[i 2 \pi \phi_{\alpha}(u)\right],
\end{aligned}
$$

where the amplitude $A_{\alpha}(u) \geqslant 0$ and the phase $\phi_{\alpha}(u)$ can be found as

$$
\begin{aligned}
\tan \left[2 \pi \phi_{\alpha}(u)\right] \\
=\frac{\int_{-\infty}^{\infty} A_{0}(y) \sin \left[\pi y^{2} \cot \alpha-2 \pi u y / \sin \alpha+2 \pi \varphi_{0}(y)\right] \mathrm{d} y}{\int_{-\infty}^{\infty} A_{0}(y) \cos \left[\pi y^{2} \cot \alpha-2 \pi u y / \sin \alpha+2 \pi \varphi_{0}(y)\right] \mathrm{d} y} .
\end{aligned}
$$

Note that $\left|F_{0}(u)\right|=A_{0}(u),\left|F_{\pi / 2}(u)\right|=A_{\pi / 2}(u), \varphi_{0}(u)$ $=\phi_{0}(u)$, and $\varphi_{\pi / 2}(u)=\phi_{\pi / 2}(u)$. In this paper we assume that $\alpha \in[0, \pi / 2]$.

The question of the importance of the phase or the amplitude in a certain transform domain is usually related to the possibility of signal reconstruction from only the phase data or only the amplitude data by applying the inverse transform. Thus we will study the operations

$$
\begin{aligned}
& f_{A, \alpha}(x)=\frac{1}{(\sin \alpha)^{1 / 2}} R^{-\alpha}\left[A_{\alpha}(u)\right](x), \\
& f_{\varphi, \alpha}(x)=\frac{1}{(\sin \alpha)^{1 / 2}} R^{-\alpha}\left\{\exp \left[i 2 \pi \varphi_{\alpha}(u)\right] \operatorname{rect}\left(\frac{u}{\Delta u}\right)\right\}(x),
\end{aligned}
$$

regarding them as signal reconstruction from the amplitude-only and phase-only data in the fractional FT domain, respectively. The rectangle function in Eq. (6) confines the phase-only function to the bandwidth $\Delta u$ of the fractional FT amplitude $A_{\alpha}(u)$. The factor $(\sin \alpha)^{-1 / 2}$ is used in Eqs. (5) and (6) for normalization.

The optical performance of the operations defined in Eqs. (5) and (6) would require a cascade of two subsystems: one, a fractional FT system ${ }^{5,10}$ from the input plane to the filtering plane and the other, an inverse fractional FT system from the filtering plane to the output plane. A spatial light modulator located in the filtering plane is programmed so that one can perform a phase filter to obtain $A_{\alpha}(u)$ or an amplitude filter to obtain $\exp \left[i 2 \pi \varphi_{\alpha}(u)\right]$. A similar optical set up was discussed in detail in Ref. 18.

We start with some examples that clearly show the dominant character of the amplitude or the phase in the fractional FT domain. It is known that the Gaussian function $\exp \left(-\pi u^{2}\right)$ is an eigenfunction of the fractional FT for any angle $\alpha$. It is a real and positive function and therefore can be reconstructed up to the constant phase factor from its fractional FT amplitude. The Gaussian function of other widths cannot be fully reconstructed by the operation of Eq. (5); nevertheless the Gaussian form of the reconstructed signal is preserved. In these cases the phase in the fractional domain does not contain information about the form of the input signal. Note that other Hermite-Gaussian functions that are also eigenfunctions of the fractional FT cannot be fully reconstructed from the knowledge of the amplitude in the fractional FT domain because they are not positive.

Let us consider as an example a chirp signal $\exp \left(i c \pi u^{2}\right)$ for which the phase in the fractional domain is certainly more important than the amplitude. Its fractional FT is also a chirp signal

$$
\begin{aligned}
& R^{\alpha}\left[\exp \left(i c \pi u^{2}\right)\right](x) \\
& \quad=\frac{\exp (i \alpha / 2)}{(\cos \alpha+c \sin \alpha)^{1 / 2}} \exp \left(i \pi u^{2} \frac{c-\tan \alpha}{1+c \tan \alpha}\right) .
\end{aligned}
$$

The signal up to the normalization factor can be reconstructed from only the phase data in the fractional domain for any $\alpha$ except a value such that $c \tan \alpha=-1$ or $\tan \alpha=c$. In the excepted cases the fractional FT of the chirp corresponds to the delta function or a constant and the amplitude information becomes dominant.

Regarding the Fourier domain, the complete signal reconstruction from the amplitude information is possible only if the signals themselves and their FTs are real, nonnegative and symmetrical. As examples we mention the following pairs: triangle signal and its FT $\operatorname{sinc}^{2} x$, selfFourier reciprocal function $\sqrt{x}$.

Note that we have considered very special functions and, in general, the complete signal is neither reconstructed from only the amplitude nor from only the phase information in the fractional FT domain. Therefore we will analyze the quality of signal restoration from its fractional FT amplitude or phase.

Because we are looking for the application of the reconstruction techniques for optical image processing we will restrict ourselves to the consideration of real nonnegative input signal. Therefore we are interested only in the amplitudes of the reconstructed signal: $\left|f_{A, \alpha}(x)\right|$ and $\left|f_{\varphi, \alpha}(x)\right|$. Then it is easy to see from Eqs. (3) and (6) that the amplitude of the signal reconstructed from only the phase information in the fractional Fourier domain can be represented as truncated inverse Fourier transform $\overparen{R^{-\pi / 2}}$ of the phase-only function

$$
\begin{aligned}
\left|f_{\varphi, \alpha}(x)\right|= & \mid \frac{1}{\sin \alpha} \exp \left(-i \pi x^{2} \cot \alpha\right) \int \operatorname{rect}\left(\frac{u}{\Delta u}\right) \\
& \times \exp \left[i \frac{2 \pi x u}{\sin \alpha}+i 2 \pi \phi_{\alpha}(u)\right] \mathrm{d} u \mid \\
= & \overparen{R^{-\pi / 2}}\left\{\exp \left[i 2 \pi \phi_{\alpha}(u \sin \alpha)\right]\right\}(x) \mid .
\end{aligned}
$$

Thus in order to understand the differences arising in the reconstruction of the signal from the phase data in the ordinary Fourier domain and in the fractional FT domain, both phase functions $\phi_{\pi / 2}(u)$ and $\phi_{\alpha}(u \sin \alpha)$ have to be compared. 


\section{SIGNAL LOCATION}

In this section we will study the question of the influence of signal shifting on its reconstruction from only the amplitude and only the phase data in the fractional Fourier domain. It is known ${ }^{17}$ that shift and modulation theorems for the fractional FT are written, respectively, as

$$
\begin{aligned}
R^{\alpha}[f(x-s)](u)= & F_{\alpha}(u-s \cos \alpha) \exp [i \pi \sin \alpha \\
& \left.\times\left(s^{2} \cos \alpha-2 s u\right)\right],
\end{aligned}
$$

$$
\begin{aligned}
& R^{\alpha}[f(x) \exp (i 2 \pi \nu x)](u) \\
& \quad=F_{\alpha}(u-\nu \sin \alpha) \exp \left[-i \pi \cos \alpha\left(\nu^{2} \sin \alpha-2 \nu u\right)\right],
\end{aligned}
$$

where $s$ and $\nu$ are constants.

For the amplitude of the reconstructed signal from only the amplitude information in the fractional Fourier domain we obtain

$$
\begin{aligned}
\left|f_{A, \alpha, s}(x)\right| & =\left|R^{-\alpha}\left[\left|F_{\alpha}(u-s \cos \alpha)\right|\right](x)\right| \\
& =\left|f_{A, \alpha, 0}\left(x-s \cos ^{2} \alpha\right)\right| .
\end{aligned}
$$

Thus the shift of the signal at $s$ produces the shift of the amplitude of the reconstructed signal at $s \cos ^{2} \alpha$. In particular this means that the amplitude of the FT does not contain information about signal localization.

If the signal is reconstructed from the phase of the fractional FT, then taking into account Eqs. (9) and (10) we observe that

$$
\begin{aligned}
\left|f_{\varphi, \alpha, s}(x)\right|= & \mid \sin ^{-1 / 2} \alpha R^{-\alpha}\left(\exp \left\{i 2 \pi \varphi_{\alpha}[u-s \cos \alpha)\right]\right\} \\
& \quad \times \exp (-2 i \pi s u \sin \alpha))(x) \mid, \\
= & \mid \sin ^{-1 / 2} \alpha R^{-\alpha}\left\{\exp \left[i 2 \pi \varphi_{\alpha}(u-s \cos \alpha)\right]\right\} \\
& \quad \times\left(x-s \sin ^{2} \alpha\right) \mid, \\
= & \mid \sin ^{-1 / 2} \alpha R^{-\alpha}\left\{\exp \left[i 2 \pi \varphi_{\alpha}(u)\right]\right\} \\
& \quad \times\left(x-s \sin ^{2} \alpha-s \cos ^{2} \alpha\right) \mid, \\
= & \left|f_{\varphi, \alpha, 0}(x-s)\right| .
\end{aligned}
$$

Therefore the operation of the signal reconstruction from only the phase data in the fractional domain is covariant with respect to the shift operation: The shift of the signal at $s$ produces the same shift $s$ of the reconstructed signal. This indicates that the corresponding optical operation is spatially invariant.

\section{SIGNAL RECONSTRUCTION FROM ITS FRACTIONAL FOURIER TRANSFORM AMPLITUDE}

We assume that $f(x)=A_{0}(x)$ is real and nonnegative. Then its FT is Hermite symmetrical with even amplitude $A_{\pi / 2}(-x)=A_{\pi / 2}(x)$ and odd phase $\varphi_{\pi / 2}(-x)=-\varphi_{\pi / 2}(x)$. This means, in particular, that the signal reconstructed from only the amplitude information in the Fourier domain has even amplitude too, which strongly decreases the size of the class of objects that could be described by only the amplitude of their FT. Moreover, as we have a

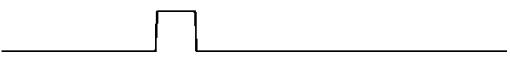

b

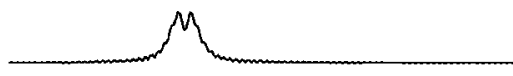

c

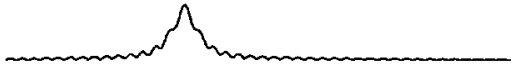

d
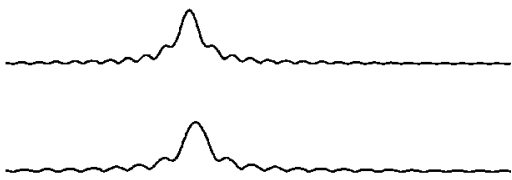

$f$

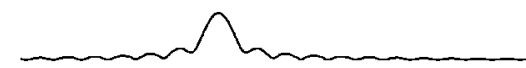

g

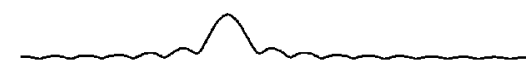

$\mathrm{h}$

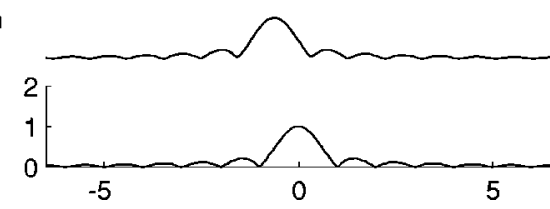

Fig. 1. Amplitude of the fractional FT of the rectangular signal for nine different values of angle $\alpha$ : a, $\alpha=0$, original signal; $\mathrm{b}$, $\alpha=\pi / 20$; c, $\alpha=\pi / 10$; d, $\alpha=3 \pi / 20$; e, $\alpha=\pi / 5$; f, $\alpha=\pi / 4$; g, $\alpha=3 \pi / 10 ; \mathrm{h}, \alpha=2 \pi / 5 ; \mathrm{i}, \alpha=\pi / 2$, amplitude of the Fourier transform.

a

b
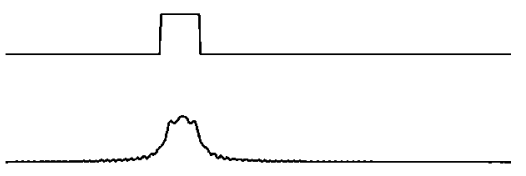

c

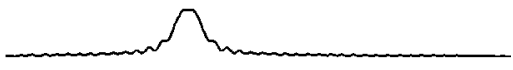

d

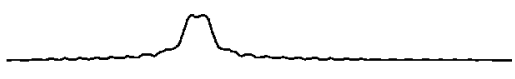

e

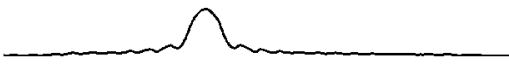

$f$

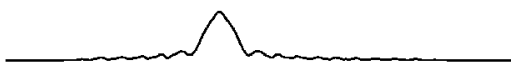

g

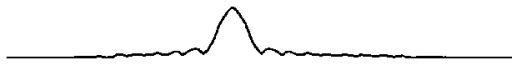

$\mathrm{h}$

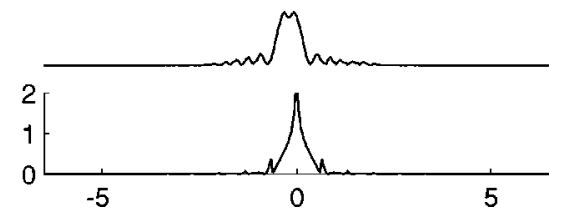

Fig. 2. Amplitude of the signal reconstructed from the amplitude of the fractional Fourier transform for the same nine values of angle $\alpha$ as in Fig. 1.

seen in the Section 3, the amplitude of the FT does not contain information about the spatial location of the signal. 
Note that the amplitude of the fractional FT of a real signal is not symmetric. Only in the case of even or odd signals is the fractional FT amplitude symmetric.

Let us consider the rectangular signal of width $d=1$ shifted from the origin of the coordinates at distance $s$ $=-1.5$. The amplitude of its fractional FT for angles $\alpha=0, \pi / 20, \pi / 10,3 \pi / 20, \pi / 5, \pi / 4,3 \pi / 10,2 \pi / 5$, and $\pi / 2$ is represented in Fig. 1a-1i, respectively. One can observe how the signal amplitude transforms itself into the symmetrical form propagating through the fractional FT system. Applying the inverse fractional FT $R^{-\alpha}$ to $\left|F_{\alpha}\right|$ we obtain the signal reconstructed from only the amplitude information in the fractional FT domain. The amplitude of the reconstructed signal is shown in Fig. 2a-2i for the angles $\alpha=0, \pi / 20, \pi / 10,3 \pi / 20, \pi / 5, \pi / 4,3 \pi / 10,2 \pi / 5, \pi / 2$, respectively. It is easy to see that the form and the size of the reconstructed signal resembles the original one only for $\alpha \leqslant \pi / 20$. Moreover in accordance with Eq. (11) the spatial location of the reconstructed signal in this region almost coincides with the location of the original one. Therefore we can conclude that in the fractional domains with relatively small angles, successful signal reconstruction from only the amplitude information is possible.

\section{SIGNAL RECONSTRUCTION FROM ITS FRACTIONAL FOURIER TRANSFORM PHASE}

It is an experimental fact that signal restoration from its FT phase results in edge enhancement. Indeed almost all edge enhancement techniques work as high-frequency passing filters. Thus methods based on the first or the second derivative of the image can be considered as filtering in the Fourier domain with the filters $u$ and $u^{2}$, respectively. These filters supress the low-frequency components and stress the higher ones. Because edges are associated with high frequencies, the application of these filters enhances edges. Under the assumption that the FT amplitude of the natural images is concentrated mainly in the low-frequency region, the filter $\left[A_{\pi / 2}(u)\right]^{-1}$ plays the role of the high-frequency passing filter. Therefore under image reconstruction from the phase of its FT, edge enhancement is usually observed.

Let us analyze this process with the example of a rectangular signal, which represents a good model for the edge investigation. We consider the rectangular signal $f(x)$ of amplitude 1 and width $d$ centered at the coordinate origin. Its FT can be written as

$$
F_{\pi / 2}(u)=\frac{\sin (\pi u d)}{\pi u} .
$$

The phase in the Fourier domain (shown in Fig. 3i) is given by

$$
g(u)=\operatorname{sgn}\left[\frac{\sin (\pi u d)}{\pi u}\right],
$$

and the inverse FT related to the signal reconstructed from only phase information of the FT is

$$
f_{\varphi, \pi / 2}(x)=\int_{-\infty}^{\infty} g(u) \exp (i 2 \pi x u) \mathrm{d} u .
$$

Note that $g(-u)=g(u)$; therefore

$$
\begin{aligned}
f_{\varphi, \pi / 2}(x)= & \int_{0}^{\infty} g(u)[\exp (i 2 \pi u x)-\exp (-i 2 \pi u x)] \mathrm{d} u \\
= & 2 i \int_{0}^{\infty} g(u) \sin (2 \pi u x) \mathrm{d} u \\
= & -\frac{i}{\pi x} \sum_{n=0}^{\infty}\left[\left.\cos (2 \pi u x)\right|_{2 n / d} ^{(2 n+1) / d}\right. \\
& \left.-\left.\cos (2 \pi u x)\right|_{(2 n+1) / d} ^{2(n+1) / d}\right] \\
= & \frac{-i\left[1-\cos \left(2 \pi \frac{x}{d}\right)\right]}{\pi x}[1] \\
& \times \sum_{n=0}^{\infty} \cos \left[2 \pi(2 n+1) \frac{x}{d}\right]
\end{aligned}
$$

It is easy to see that for $x_{k}=k d$, where $k$ is an integer, $f_{\varphi, \pi / 2}\left(x_{k}\right)=0$. Meanwhile for $x_{l}=d(l+1 / 2)$ the modulus $\left|f_{\varphi, \pi / 2}(x)\right|$ has local maxima. Moreover the absolute maxima are observed for $l=0$ and $l=-1$, which correspond to the borders of the rectangular signal $x=-d / 2$ and $x=d / 2$. Other maxima are related to the principal ones as $\left|f_{\varphi, \pi / 2}[d(l+1 / 2)]\right|=1 /|2 l+1|\left|f_{\varphi, \pi / 2}(d / 2)\right|$. The amplitude of the signal reconstructed from phase information in the Fourier domain is represented in Fig. 4i. The clear indication of the edges of the rectangular signal is observed. In the meantime, the additional local maxima at the points $x_{l}=d(l+1 / 2)$, which relate to the quasi-periodical structure of $g(u)$, can lead to confusing

a

b

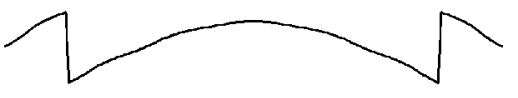

c

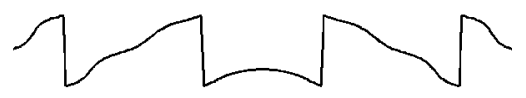

d

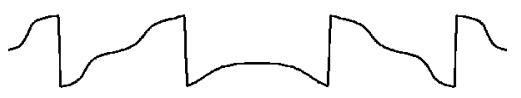

e

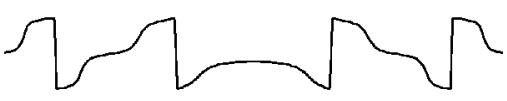

$f$

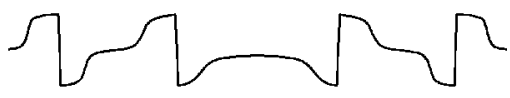

g

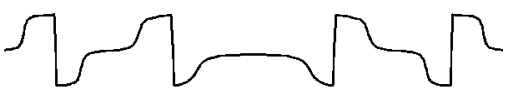

$\mathrm{h}$
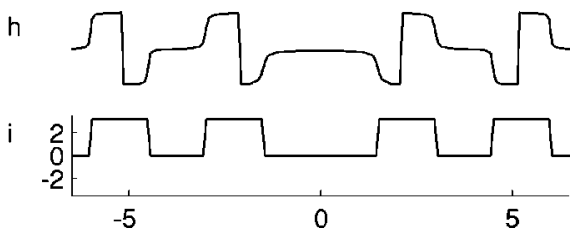

Fig. 3. Phase profile in the Fourier domain for the rectangular signal, simulating the effect of the signal reconstruction from the phase of its fractional FT for the same nine values of angle $\alpha$ as in Fig. 1. 


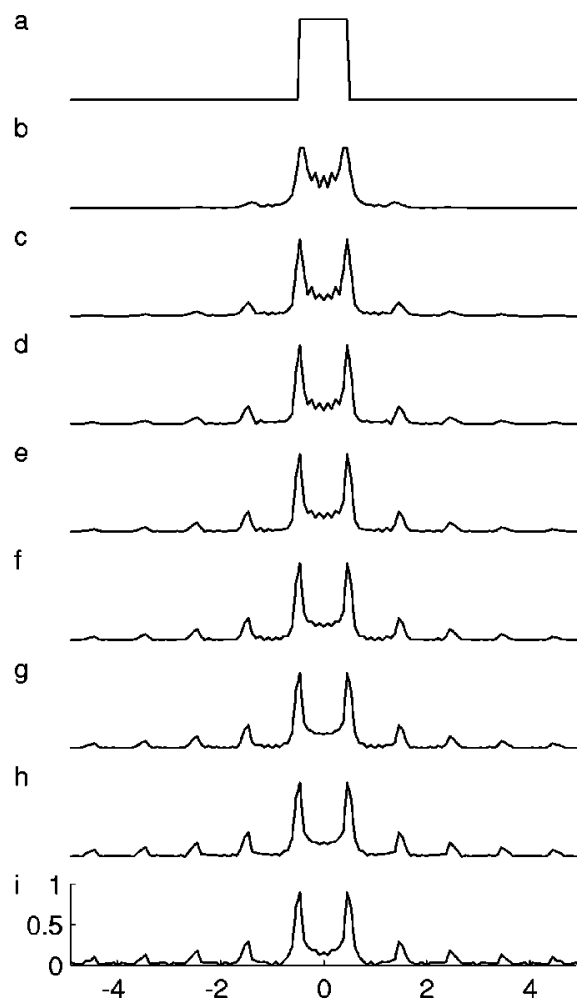

Fig. 4. Amplitude of the signal reconstructed from the phase of the fractional FT for the same nine values of angle $\alpha$ as in Fig. 1.

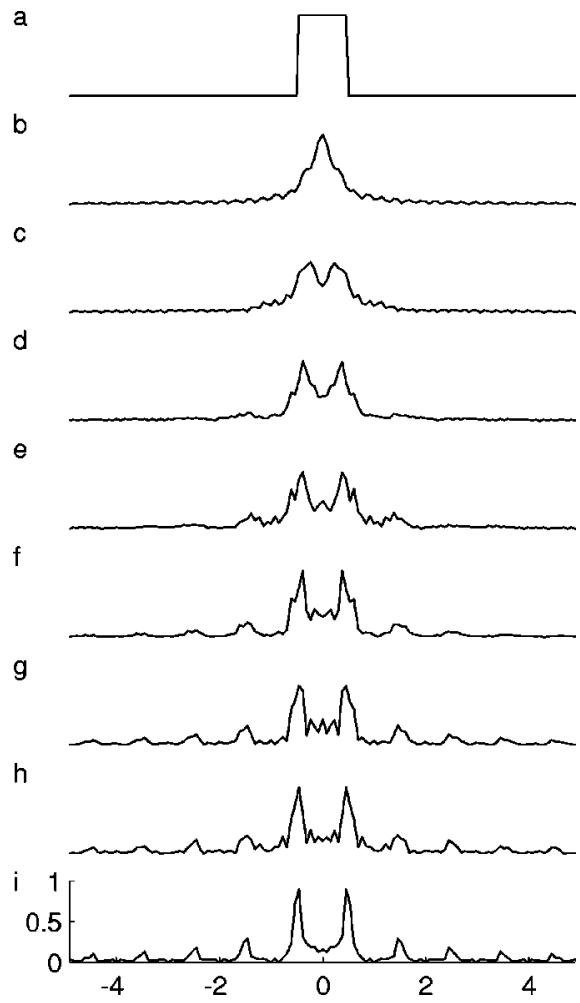

Fig. 5. Amplitude of the signal reconstructed from the phase of the fractional FT, taken in the region where the amplitude of the fractional FT is relatively large, for the same nine values of angle $\alpha$ as in Fig. 1. conclusions if a more complicated signal is considered. Therefore the choice of the correct threshold for edge detection by the implementation of signal restoration from the FT phase is important and has to be defined before any reconstruction operation. As we will see in Section 6 in the case of the real-world images, the additional local maxima interfere among themselves, producing the noisy background of the image reconstructed from its FT phase.

Let us now consider what features of the rectangular signal can be revealed from only the phase information in the fractional Fourier domain. The fractional FT of the rectangular signal $f(x)$ of amplitude 1 and width $d$ centered at the coordinate origin is given by

$$
\begin{aligned}
F_{\alpha}(u)= & \frac{\exp (i \alpha / 2)}{(i \sin \alpha)^{1 / 2}} \exp \left(i \pi u^{2} \cot \alpha\right) \\
& \times \int_{-d / 2}^{d / 2} \exp \left(i \pi \frac{x^{2} \cos \alpha-2 x u}{\sin \alpha}\right) \mathrm{d} x, \\
= & \frac{\exp (i \alpha / 2)}{(i \sin \alpha)^{1 / 2}} \exp \left(i \pi u^{2} \cot \alpha\right) A_{\alpha}(u) \exp \left[i 2 \pi \phi_{\alpha}(u)\right], \\
= & \frac{\exp (i \alpha / 2)}{(i \sin \alpha)^{1 / 2}} \exp \left(i \pi u^{2} \cot \alpha\right) h_{\alpha}(u),
\end{aligned}
$$

where

$$
\begin{aligned}
h_{\alpha}(u) & =\int_{-d / 2}^{d / 2} \exp \left(i \pi \frac{x^{2} \cos \alpha-2 x u}{\sin \alpha}\right) \mathrm{d} x \\
& =\exp \left[-i \frac{2 \pi u^{2}}{\sin (2 \alpha)}\right] \int_{-d / 2}^{d / 2} \exp \left[i \pi \frac{(x-u / \cos \alpha)^{2}}{\tan \alpha}\right] \mathrm{d} x .
\end{aligned}
$$

Note that the integral in the last term of Eq. (18) is a typical integral arising under consideration of the scalar paraxial diffraction by a rectangular aperture (see, for example, Refs. 2 and 3). Based on Eqs. (7.4.38) and (7.4.39) from Ref. 19, and representing this integral through the Fresnel integrals

$$
\begin{aligned}
& C(z)=\int_{0}^{z} \cos \left(\frac{\pi}{2} t^{2}\right) \mathrm{d} t, \\
& S(z)=\int_{0}^{z} \sin \left(\frac{\pi}{2} t^{2}\right) \mathrm{d} t,
\end{aligned}
$$

we obtain

$$
h_{\alpha}(u)=[(\tan \alpha) / 2]^{1 / 2} \exp [-i \beta(u)](\Delta C+i \Delta S),
$$

where

$$
\begin{aligned}
& \Delta C=C\left(z_{2}\right)-C\left(z_{1}\right), \\
& \Delta S=S\left(z_{2}\right)-S\left(z_{1}\right),
\end{aligned}
$$

$\beta(u)=2 \pi u^{2} / \sin (2 \alpha), \quad z_{1}=(-d \cos \alpha-2 u) /[\sin (2 \alpha)]^{1 / 2}$, and $z_{2}=(d \cos \alpha-2 u) /[\sin (2 \alpha)]^{1 / 2}$. Thus the amplitude $A_{\alpha}(u)$ and the phase $2 \pi \phi_{\alpha}(u)=\arg h_{\alpha}(u)$ of $h_{\alpha}(u)$ are given by

$$
\begin{aligned}
A_{\alpha}(u) & =\left\{\frac{\tan \alpha}{2}\left[(\Delta C)^{2}+(\Delta S)^{2}\right]\right\}^{1 / 2}, \\
2 \pi \phi_{\alpha}(u) & =\arctan (\Delta S / \Delta C)-\beta(u) .
\end{aligned}
$$


As we have seen in Section 2 the amplitude of the signal reconstructed from only the phase data in the fractional FT domain is determined by the modulus of the truncated inverse FT of function $\exp \left[i 2 \pi \phi_{\alpha}(u \sin \alpha)\right]$ [see Eq. (8)]. As shown in Appendix A [see Eq. (A4)] the phase $2 \pi \phi_{\alpha}(u \sin \alpha)$ can be expressed through the auxiliary functions $\mathfrak{f}(z)$ and $\mathfrak{g}(z)^{19}$ as

$$
2 \pi \phi_{\alpha}(u \sin \alpha)=\left(\pi d^{2} \cot \alpha\right) / 4+\eta(d, \alpha, u),
$$

where

$\tan [\eta(d, \alpha, u)]$

$$
=\frac{\cos \gamma\left[\mathfrak{f}\left(y_{1}\right)-\mathfrak{f}\left(y_{2}\right)\right]+\sin \gamma\left[\mathfrak{g}\left(y_{1}\right)+\mathfrak{g}\left(y_{2}\right)\right]}{\cos \gamma\left[\mathfrak{g}\left(y_{1}\right)-\mathfrak{g}\left(y_{2}\right)\right]-\sin \gamma\left[\mathfrak{f}\left(y_{1}\right)+\mathfrak{f}\left(y_{2}\right)\right]},
$$

$\gamma=\pi u d$, and $y_{1,2}=\sqrt{2}(\mp d \sqrt{\cot \alpha} / 2-u \sqrt{\tan \alpha})$.

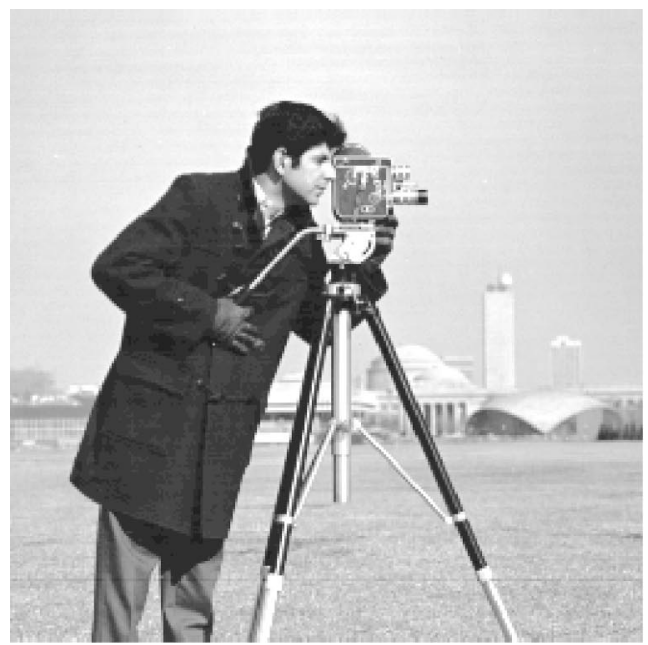

(a)

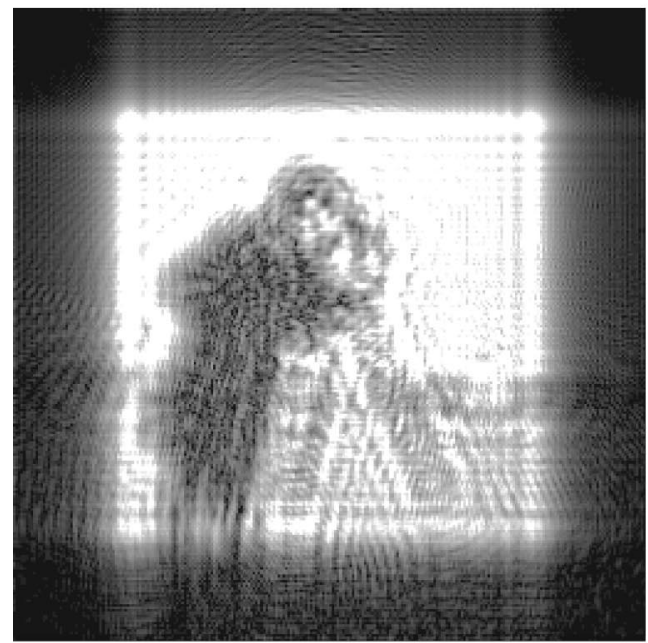

(c)
Because the first term of the sum on the right-hand side of Eq. (22) does not depend on $u$, only the phase function $\eta(d, \alpha, u)$ defines the differences arising in the signal reconstructed from the phase of the fractional FTs of different orders. Let us analyze the behavior of $\eta(d, \alpha, u)$ for different $\alpha$. In Fig. 3a-3i the phase $\eta(1, \alpha, u)$ for $\alpha=0, \pi / 20, \pi / 10,3 \pi / 20, \pi / 5, \pi / 4,3 \pi / 10$, $2 \pi / 5$, and $\pi / 2$, respectively, is presented. It is easy to see that the form of the phase for all angles (except perhaps $\alpha \leqslant \pi / 20$ ) has similar structure: It is quasi-periodic with the same period outside the central region, whose size $r(\alpha)$ increases for small $\alpha$.

The amplitude of the signal reconstructed from the phase of the fractional FT [see Eq. (8)] is defined as

$$
\left|f_{\varphi, \alpha}(x)\right|=\left|\int \operatorname{rect}\left(\frac{u}{\Delta u}\right) \exp [i \eta(d, \alpha, u)+i 2 \pi x u] \mathrm{d} u\right|,
$$

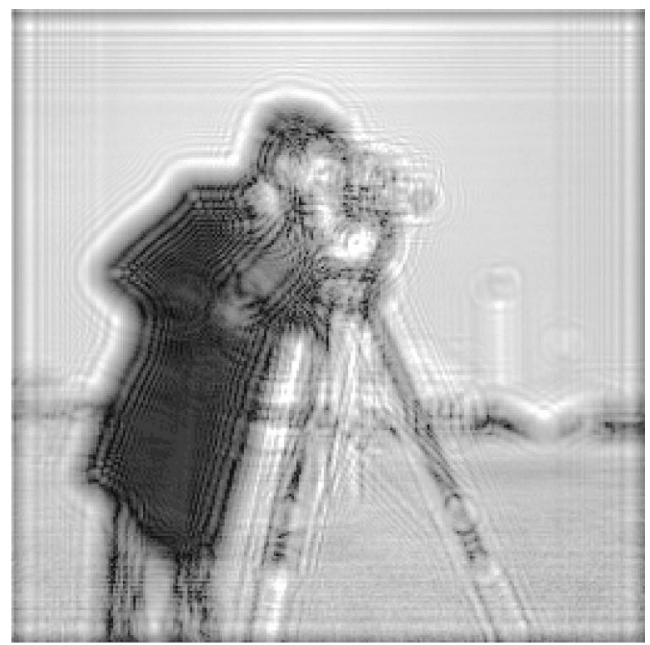

(b)

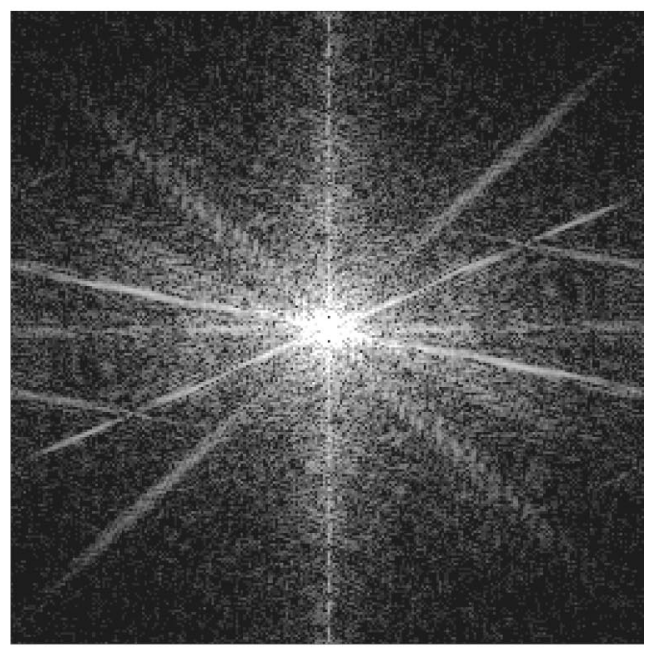

(d)

Fig. 6. Amplitude of the fractional FT of the cameraman image for different values of angle $\alpha$ : a, $\alpha=0$, original signal; b, $\alpha=\pi / 20 ; \mathrm{c}, \alpha=\pi / 4 ; \mathrm{d}, \alpha=\pi / 2$, amplitude of the FT. 


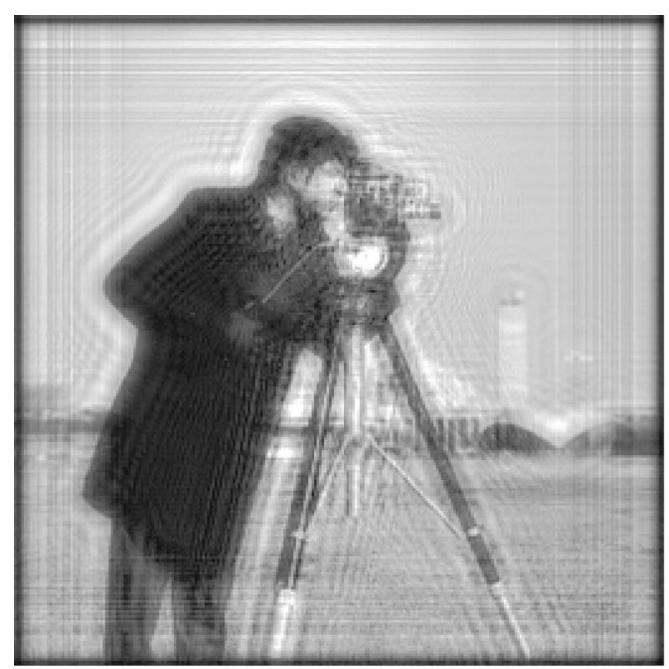

(a)

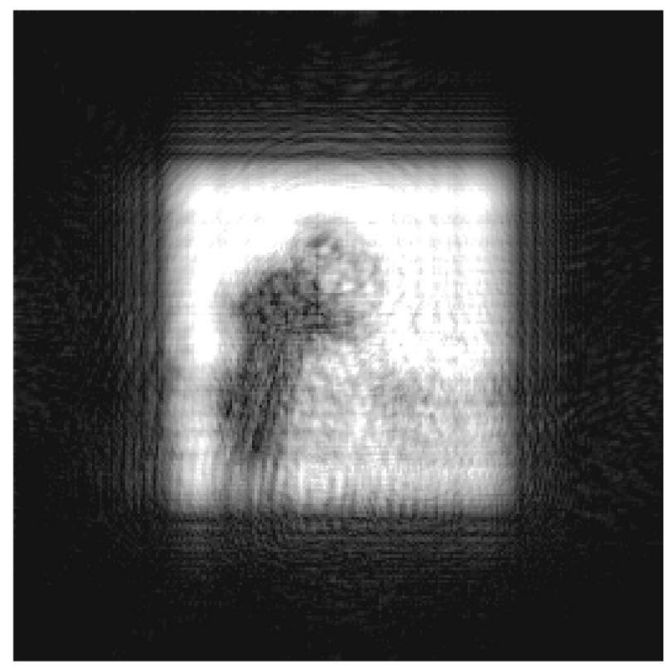

(c)

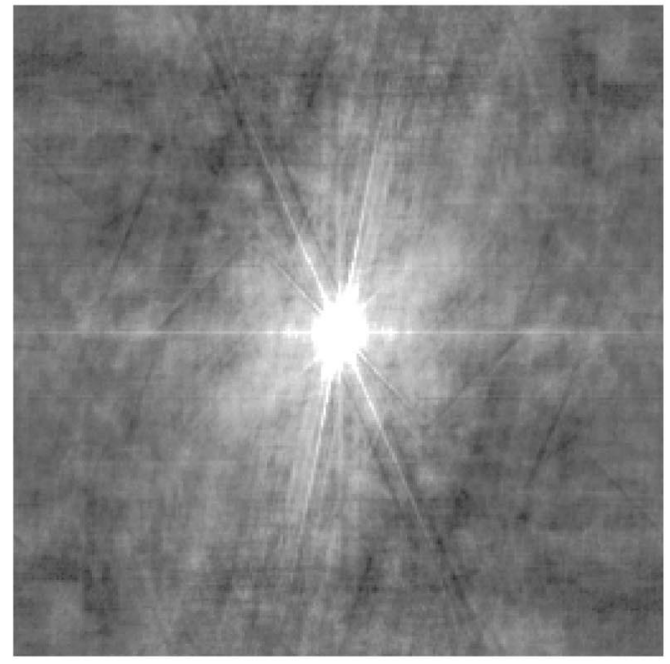

(e)

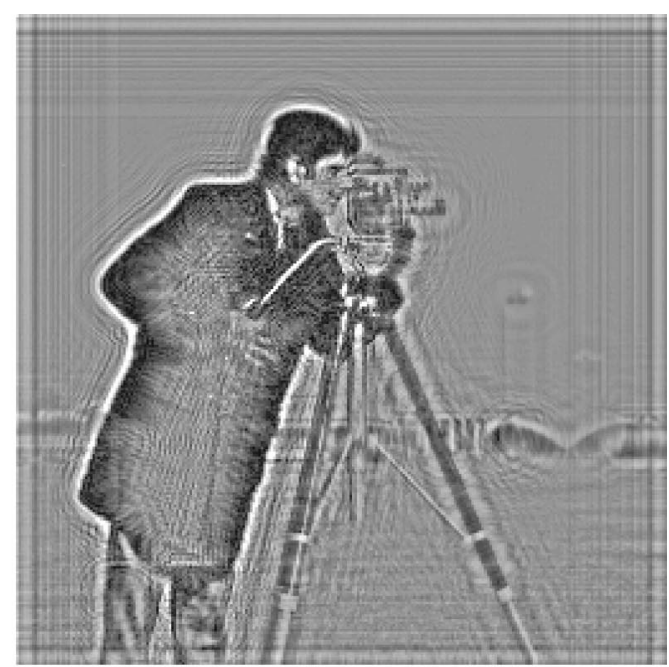

(b)

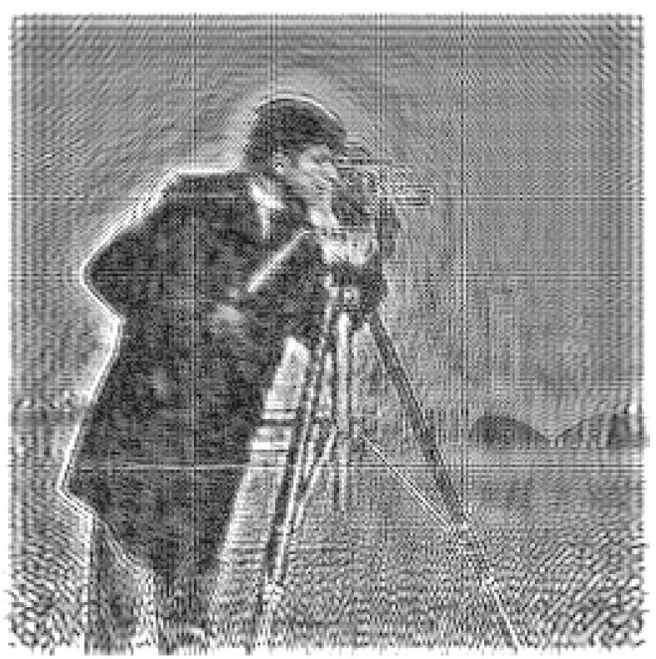

(d)

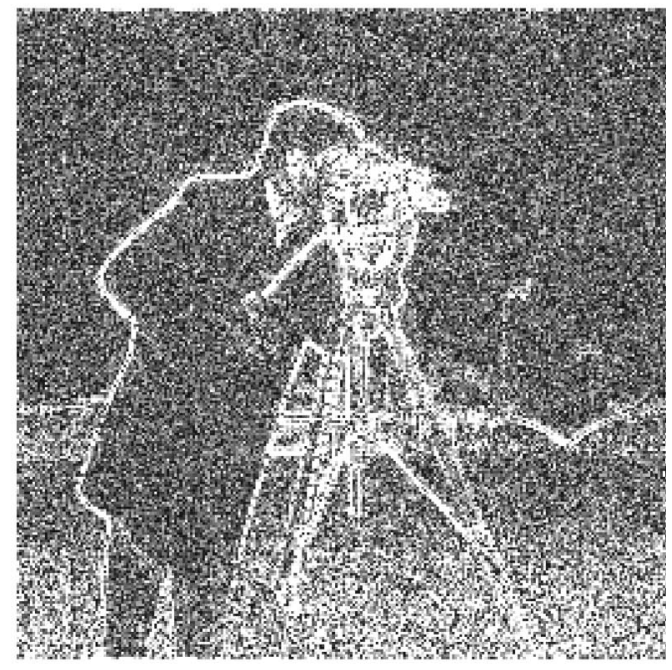

(f)

Fig. 7. Amplitude of the image reconstructed from (a, c, e) the amplitude-only data and (b, $d, f)$ the phase-only data of the fractional FT of the cameraman image for different values of angle $\alpha$ : (a, b) $\alpha=\pi / 20$, (c, d) $\alpha=\pi / 4$, (e, f) $\alpha=\pi / 2$, FT domain. 
where the rectangle function confines the phase-only function to the bandwidth $\Delta u$ of the fractional FT amplitude $A_{\alpha}(u / \sin \alpha)$. In general $\Delta u$ depends on the width $d$ of the rectangular signal and $\alpha$. It can be defined as the region outside of which $A_{\alpha}<c$, where $c$ is a threshold depending on the accuracy of the measurements or the calculation of $A_{\alpha}$. If we assume that phase information is available for a very wide region $\left[c=1 \% \max \left(A_{\alpha}\right)\right]$ the signal edges can be successfully reconstructed from the phase data in almost all fractional domains (see Fig. 4a4i). We also note that the higher the angle of the fractional FT, the smaller the energy of the reconstructed signal concentrated inside the area defined by its edges. Additional quasi-periodic peaks appear outside of this region. The period of the peaks equals the width of the rectangle.

In practice phase information is known only in the regions where its amplitude has significant values. The signal reconstructed from the phase information of its fractional FT in the region $\Delta u$ defined by threshold $c$ $=10 \% \max \left(A_{\alpha}\right)$ is represented in Figs. 5a-5i for the angles $\alpha=0, \pi / 20, \pi / 10,3 \pi / 20, \pi / 5, \pi / 4,3 \pi / 10,2 \pi / 5$, and $\pi / 2$, respectively. One can observe that in this case the range of angles for which signal edges can be successfully reconstructed moves toward higher values. Thus for $\alpha<\pi / 10$ any information about the signal size is ambiguous.

\section{IMAGE RECONSTRUCTION FROM ONLY AMPLITUDE DATA AND FROM ONLY PHASE DATA IN THE FRACTIONAL FOURIER DOMAIN}

In this section we consider the reconstruction of the realworld image from only the amplitude data and from only the phase data in the fractional FT domains. The original image is a photograph of a cameraman taken from MATLAB® archives. It contains $256 \times 256$ pixels and is shown in Fig. 6a. The program for the calculation of the two-dimensional fractional FT is based on the algorithm proposed in Refs. 10 and 20 . Figures $6 \mathrm{~b}-6 \mathrm{~d}$ represent the moduli of the fractional FT of the image for the angles $\pi / 20, \pi / 4$, and $\pi / 2$, respectively.

In Fig. 7 the images reconstructed from the amplitude (a, c, e) and phase (b, d, f) information of the FT for angles $\alpha=\pi / 20$ (Figs. 7a and 7b), $\alpha=\pi / 4$ (Figs. 7c and 7d), and $\alpha=\pi / 2$ (Figs. 7e and 7f) are shown. We observe that for small angles, significant information about the original image can be obtained from both the amplitude-only data (Fig. 7a) and phase-only (Fig. 7b) data. In accordance with Eq. (11) the image reconstructed from the amplitude data is slightly shifted toward the center with respect to the original one. Comparing, for example, the figure of the cameraman in Figs. $7 \mathrm{a}$ and $7 \mathrm{~b}$ we notice that the reconstruction from the phase data is better for highcontrast details while the low-contrast objects such as the tower are reconstructed better from the amplitude data.

Increasing the angle of the fractional FT, we observe that the reconstruction from the amplitude data rapidly loses information about the original image. Thus in Fig. $7 \mathrm{c}(\alpha=\pi / 4)$ there is only the barest indication of the cameraman. However, the image reconstructed from the phase data still contains significant information about the original image. In Fig. 7d, despite the fact that some details such as the buildings or the pleats on the trousers cannot be distinguished, the figure and the face of the cameraman and some components of the camera can be seen clearly.

The amplitude of the FT does not permit reconstruction of any significant details of the original image (see Fig. 7e). However, the image reconstructed from the FT phase (Fig. 7f) clearly shows the edges of the original image. Just as rectangular signal reconstruction from the FT phase eliminates information about the intensity levels of the original image, and almost binarize it, the additional peaks interfering with each other produce the noisy background (a statistical noise). Note that the presence of the background noise does not significantly degrade the edge detection.

\section{CONCLUSIONS AND DISCUSSIONS}

We have studied the question of the importance of the amplitude and the phase information in the fractional FT domain for the case of real positive signals using the examples of the rectangular signal and a real-world image. We have shown that the signal reconstructed from the amplitude of the fractional FT for small angles reveals significant details of the original signal. This facility drastically decreases with increasing angle. On the other hand, phase information in the fractional FT domain for all angles (except perhaps the smallest ones) almost always (except for some signals with special symmetry) permits reconstruction of the main characteristics of the original signal. Approaching the angle $\pi / 2$, the reconstructed image shows almost binary structure in which the edges of the original image are indicated by local maxima of intensity.

In this paper we have considered the image reconstruction from the amplitude or phase of its fractional FT of the same order for both transversal coordinates. We think that in the case of the anamorphic fractional FT the signal reconstruction from the amplitude-only data will be sufficiently good if both orders are small. Regarding the image reconstruction from the phase of the fractional FT, we believe that the main details of the images can be observed even if there is a significant difference between the fractional orders, except for the case of the zeroth order for one of the directions. Nevertheless, the vertical and horizontal edges of the reconstructed image can be enhanced with a different contrast, depending on the corresponding orders of the anamorphic fractional FT. This is a topic for further research.

\section{APPENDIX A}

Alternatively, based on the representation of the Fresnel integrals through the auxiliary functions $\mathfrak{f}(z)$ and $\mathfrak{g}(z)^{19}$

$$
\begin{aligned}
& C(z)=\frac{1}{2}+\mathfrak{f}(z) \sin \left(\frac{\pi}{2} z^{2}\right)-\mathfrak{g}(z) \cos \left(\frac{\pi}{2} z^{2}\right), \\
& S(z)=\frac{1}{2}-\mathfrak{f}(z) \cos \left(\frac{\pi}{2} z^{2}\right)-\mathfrak{g}(z) \sin \left(\frac{\pi}{2} z^{2}\right),
\end{aligned}
$$


and after the derivation

$$
\begin{aligned}
\Delta C+ & i \Delta S \\
= & \left\{\mathfrak{f}(z) \sin \left(\frac{\pi}{2} z^{2}\right)-\mathfrak{g}(z) \cos \left(\frac{\pi}{2} z^{2}\right)\right. \\
& \left.-i\left[\mathfrak{f}(z) \cos \left(\frac{\pi}{2} z^{2}\right)+\mathfrak{g}(z) \sin \left(\frac{\pi}{2} z^{2}\right)\right]\right\}_{z_{1}}^{z_{2}} \\
= & -\left\{\exp \left(i \frac{\pi}{2} z^{2}\right)[\mathfrak{g}(z)+i \mathfrak{f}(z)]\right\}_{z_{1}}^{z_{2}} \\
= & \exp \left[i \frac{\pi}{4}\left(z_{1}^{2}+z_{2}^{2}\right)\right]\left\{\exp \left[i \frac{\pi}{4}\left(z_{1}^{2}-z_{2}^{2}\right)\right]\left[\mathfrak{g}\left(z_{1}\right)+i \mathfrak{f}\left(z_{1}\right)\right]\right. \\
& \left.-\exp \left[-i \frac{\pi}{4}\left(z_{1}^{2}-z_{2}^{2}\right)\right]\left[\mathfrak{g}\left(z_{2}\right)+i \mathfrak{f}\left(z_{2}\right)\right]\right\},
\end{aligned}
$$

where

$$
\begin{aligned}
& \frac{\pi}{4}\left(z_{1}^{2}+z_{2}^{2}\right)=\pi \cot \alpha \frac{d^{2}}{4}+\frac{2 \pi u^{2}}{\sin (2 \alpha)}=\pi \cot \alpha \frac{d^{2}}{4}+\beta(u), \\
& \frac{\pi}{4}\left(z_{1}^{2}-z_{2}^{2}\right)=\pi \frac{u d}{\sin \alpha},
\end{aligned}
$$

we can rewrite Eq. (20) for $h_{\alpha}(u)$ as

$$
\begin{aligned}
h_{\alpha}(u)= & {[(\tan \alpha) / 2]^{1 / 2} \exp \left[i \pi \frac{d^{2} \cot \alpha}{4}\right]\left\{\exp \left[i \pi \frac{u d}{\sin \alpha}\right]\right.} \\
& \times\left[\mathfrak{g}\left(z_{1}\right)+i \mathfrak{f}\left(z_{1}\right)\right]-\exp \left[-i \pi \frac{u d}{\sin \alpha}\right] \\
& \left.\times\left[\mathfrak{g}\left(z_{2}\right)+i \mathfrak{f}\left(z_{2}\right)\right]\right\} .
\end{aligned}
$$

Despite Eq. (20) being more compact, Eq. (A4) is preferable for numerical calculations. Thus the auxiliary functions $\mathfrak{f}(z)$ and $\mathfrak{g}(z)^{19}$ are approximated for $z \geqslant 0$ and $|\epsilon(z)| \leqslant 2 \times 10^{-3}$ as

$$
\begin{aligned}
\mathfrak{f}(z) & =\frac{1+0.926 z}{2+1.792 z+3.104 z^{2}}+\epsilon(z), \\
\mathfrak{g}(z) & =\frac{1}{2+4.142 z+3.492 z^{2}+6.670 z^{3}}+\epsilon(z),
\end{aligned}
$$

and the relationships

$$
\begin{aligned}
\mathfrak{f}(z)+\mathfrak{f}(-z) & =\cos \left(\frac{\pi}{2} z^{2}\right)-\sin \left(\frac{\pi}{2} z^{2}\right), \\
\mathfrak{g}(z)+\mathfrak{g}(-z) & =\cos \left(\frac{\pi}{2} z^{2}\right)+\sin \left(\frac{\pi}{2} z^{2}\right),
\end{aligned}
$$

permit calculation of the functions $\mathfrak{f}(z)$ and $\mathfrak{g}(z)$ for negative $z$.

\section{ACKNOWLEDGMENTS}

Financial assistance from the Spanish Ministry of Science and Technology (project TIC 2002-01846) is acknowledged. T. Alieva acknowledges the financial support of Secretaría de Estado de Educación y Universidades de España (SB2000-0166) (Spanish Ministry of Education, Culture, and Sports).

T. Alieva may be reached by e-mail at talieva @fis.ucm.es.

\section{REFERENCES}

1. J. L. Horner and P. D. Gianino, "Phase-only matched filtering," Appl. Opt. 23, 812-816 (1984).

2. A. Vanderlugt, Optical Signal Processing (Wiley, New York, 1992).

3. G. O. Reynolds, J. B. DeVelis, G. B. Parrent, and B. J. Thompson, The New Physical Optics Notebook: Tutorials in Fourier Optics (SPIE Press, Bellingham, Wash., 1989).

4. D. Mendlovic, H. M. Ozaktas, and A. W. Lohmann, "Fractional correlation," Appl. Opt. 34, 303-309 (1995).

5. D. Mendlovic, Y. Bitran, R. G. Dorsch, and A. Lohmann, "Optical fractional correlation: experimental results," J. Opt. Soc. Am. A 12, 1665-1670 (1995).

6. J. García, D. Mendlovic, Z. Zalevsky, and A. Lohmann, "Space variant simultaneous detection of several objects using multiple anamorphic fractional Fourier transform filters," Appl. Opt. 35, 3945-3952 (1996).

7. J. Garcia, R. G. Dorsch, A. W. Lohmann, C. Ferreira, and Z. Zalevsky, "Flexible optical implementation of fractional Fourier processors: application to correlation and filtering," Opt. Commun. 133, 393-400 (1997).

8. O. Akay and G. F. Boudreaux-Bartels, "Fractional convolution and correlation via operator methods and an application to detection of linear FM signals," IEEE Trans. Signal Process. 49, 979-993 (2001).

9. T. Alieva and M. L. Calvo, "Generalized fractional convolution," in Perspectives in Modern Optics and Optical Instrumentation (Anita Publications, New Delhi, 2002), pp. 282292.

10. H. M. Ozaktas, Z. Zalevsky, and M. A. Kutay, The Fractional Fourier Transform with Applications in Optics and Signal Processing (Wiley, New York, 2001).

11. A. Kozma and D. Kelly, "Spatial filtering for detection of signals submerged in noise," Appl. Opt. 4, 387-392 (1965).

12. A. V. Oppenheim and J. S. Lim, "The importance of phase in signals," Proc. IEEE 69, 529-541 (1981).

13. L. B. Lesem, P. M. Hirsch, and J. A. Jordan, "The kinoform: a new wavefront reconstruction device," IBM J. Res. Dev. 13, 150-155 (1969).

14. A. W. Lohmann, D. Mendlovic, and G. Shabtay, "Significance of phase and amplitude in the Fourier domain," J. Opt. Soc. Am. A 14, 2901-2904 (1997).

15. D. Mendlovic and H. M. Ozaktas, "Fractional Fourier transforms and their optical implementation: I," J. Opt. Soc. Am. A 10, 1875-1881 (1993).

16. H. M. Ozaktas and D. Mendlovic, "Fractional Fourier transformations and their optical implementation: II," J. Opt. Soc. Am. A 10, 2522-2531 (1993).

17. L. B. Almeida, "The fractional Fourier transform and timefrequency representations," IEEE Trans. Signal Process. 42, 3084-3091 (1994).

18. T. Alieva, M. L. Calvo, and M. J. Bastiaans, "Power filtering of n-order in the fractional Fourier domain," J. Phys. A 35, 7779-7785 (2002).

19. M. Abramovich and I. A. Segun, Handbook of Mathematical Functions (Dover, New York, 1965).

20. H. M. Ozaktas, O. Arikan, M. A. Kutay, and G. Bozdagi, "Digital computation of the fractional Fourier transform," IEEE Trans. Signal Process. 44, 2141-2150 (1996). 